\title{
A numerical method for the solution of an autonomous initial value problem
}

\author{
FLAVIUS PĂTRULESCU
}

\section{ABSTRACT.}

Using a known interpolation formula we introduce a class of numerical methods for approximating the solutions of scalar initial value problems for first order differential equations, which can be identified as explicit Runge-Kutta methods. We determine bounds for the local truncation error and we also compare the convergence order and the stability region with those for explicit Runge-Kutta methods, which have convergence order equal with number of stages (i.e. with 2, 3 and 4 stages). The convergence order is only two, but our methods have a larger absolute stability region than the above mentioned methods. In the last section a numerical example is provided, and the obtained numerical approximation is compared with the corresponding exact solution.

Tiberiu Popoviciu InStitute of Numerical ANALYSiS

ROMANIAN ACADEMY

P.O. BOX 68-1, 400110 CLUJ-NAPOCA, ROMANIA

UNIVERSITY BABEŞ-BOLYAI

400084 CLUJ-NAPOCA, ROMANIA

E-mail address: fpatrulesculictp.acad.ro 